\title{
EL PRIMER DESCUBRIMIENTO DE AMERICA
}

Antonio Lezama Astigarraga *

\section{INTRODUCCIÔN}

¿Oue ves, hijo? - Bl paraiso, padre.

Tales deben de haber sido las palabras, hace unos doce mil años, de los primeros hombres que divisaron las inmensas praderas cubiertas de animales que se extendian al sur del Saskatchewan tras largos milenios de duro peregrinaje por las heladas estepas de Siberia y Alaska.

A partir de esse momento, América, sobre la que ya caminaban, sin transición aparente, desde sus origenes siberianos, cobrará un caráter propio estimulando, con sus nuevas e infinitas posibilidades, de tal modo a sus primetos pobladores que, en el milenio siguiente, realizarán la proeza de poblarla hasta sus más remotos rincones.

Sobre esta aventura colonizadora (sin duda la más extraordinaria de la Historia), sus probables causas, sus mecanismos y sus consecuencias para los indoamericanos, tratarem en el presente articulo.

Sobre el poblamiento primitivo de América se han escrito infiaidad de trabajos a lo largo del siglo, fundamentalmente en lengua inglesa. Se ha enfocado el tema desde diversos ángulos: la antiguedad de determinados

\footnotetext{
- Doutorado em Arqueologia na Escala de Altos Estudos em Ciencias Sociais, Parin, França. Professoor do Departamento de Antropologia, Faculdad Humanidedes y Cieacias de la Fidacación, Urugazy.
} 
hallazgos arqueologicos, las diferencias o similitudes de las razas amerindias, las probables vias de aceso al continente, las asociaciones entre los rasgos arqueológicos e entnográficos de América y del Viejo Mundo, la lingüistica, la genética, etc.

Creemos que a esta altura del desarrollo de las investigaciones es posible realizar una síntesis que aclare la interrelación de los diferentes aspectos que entraron en juego para provocar el poblamiento del Nuevo Mundo.

Esta sintesis se basa en un paradigma de caráter universal el cual, a nuestro juicio, sienta una base definitiva para la comprensión del problema del poblamiento.

Dicho paradigma consiste en que el poblamiento de América no fue un fenómeno excepcional donde lis reglas normales del comporlamiento humano se vieram completamente alteradas. Esto abriria el campo a las hipótesis más diversas e inverosímiles. Recordemos sino la llegada de australianos vía la Antártida, o el arribo de elementos "arios" generadores de las "altas culturas", entre tantas otras (Canals Frau 1973:498-500; Rivet 1960; 106-111).

El poblamiento de América es un hecho único pero responde a las pautas de esse comportamiento "normal".

Definir dicho comportamiento será la clave del presente trabajo, este es el que explica toda la aventura colonizadora del género humano, no sólo la americana, sino tambiém la del Viejo Mundo.

El poblamiento de América no será visto entonces como un acontecimiento extraordinario, sino como el filtimo capitulo del poblamiento de la tierra que comicnza en los origenes africanos de $1^{*}$ humanidad.

Mucho se ha discutido $y$ se discute sobre la fecha del primer descumprimiento de América y será la arqueologia la que en definitiva, por acumulạtión de evidencias, zanje la polémica (Meltzer 1989, Laming Emperaire 1980, Martin 1973, Bray 1988, Laughlin 1977, Klein 1975, Haynes 1988, Dillebay 1988, Graham \& Heizer 1968, Griffin 1976, Jelinek 1971, Diamond 1987, Rouse 1976).

La interpretación tradicional del problema del poblamiento de América fue la difusionista, que tan fuertemente marcó a la historiografia uruguaya. 
Las concepciones difusionistas surgieron y se desarrollaron entre las décadas del 30 y del 50 . Su expoaente principal es la obra del etnólogo francés Paul Rivet Los origenes del hombre americano, publicada por primeira vez en 1943 y revisada en 1957.

Las ideas difusionistas encontraron un medio favorable eatre los investigadores argentinos quienes agregaron sus propios puntos de vista (Imbelloni 1948), los que, con la obra de S. Canals Frau Prehistoria de América (publicada en 1950 y re-editada hasta 1973) alcanzaron amplia difusión en el Uruguay.

La concepción difusionista esquemáticamente puede reducirse al principio que las grandes innovaciones de la humanidad se habrian producido en algunos pocos puntos privilegiados del planeta. Desde alli se difundirian, directa o indirectamente, a los demás pueblos del mundo.

De acuerdo a este marco teórico - y en razón de una supuesta supremacia histórica del Viejo Mundo - los rasgos culturales, antropométricos, lingúísticos, ete. presentes en el Nuevo Mundo deben tener su origen en el "Viejo". El corolario es un conjunto de demostraciones basadas en analogias.

Si en América encontramos un rasgo A, por ejemplo la pesca envenenando las aguas, este tiene su origen en un comportamiento similar en el Viejo Mundo que pasa a ser el rasgo $\mathrm{A}^{*}$; luego la tarea consisten en establecer la probabler ruta de migración de $\mathrm{A}^{\prime}$ hasta $\mathrm{A}$.

Patra establecer la lista de rasgos $\mathrm{A}$ y $\mathrm{A}^{\prime}$ se recurrió a todo tipo de elementos, aunque el acento estuvo puesto especialmente en las caracteristicas antropológicas (fisicas) de los iadigenas. Esto fue estimulado por la aparente diversidad de razas americanas, En segundo lugar se apela a una comparación de las lenguas (también caracterizadas por una gran variedad); y por último al registro arqueológico.

La crítica definitiva vendrá de los propios discipulos de Paul Rivet, del Museo del Hombre de Paris. Sus principales aspectos se encuentran resumidos en lat obra de A. Laming Emperaire El problema de los origenes americanos, Tearias, hipótesis y documentos publicada en forma póstuma en 1980.

La falta de sistematización del análisis y la pobreza del registro arqueológico llamado a sustentar las hipotesis, hace que el difusionísmo sea abandonado como paradigma válido para la interpretación del poblamiento da América. 
Su error fundamental consistio en no considerar que este era un problema histórico (́prehistórico) y que los dalos para su solución debian, necesariamente, ser aportados por la arqueologia. El ticmpo es la variable fundamental en todo processo histórico y el difusionismo fracasó cuando fue demostrado que los rasgos similares encontrados en ambos "mundos", estaban cronológicamente separados por milenios, Fracasó también en no considerar que determinado rasgo cultural es el fruto de una realidad histórica concreta y que su conscrvación, abandono o transformación, debe ser explicado en base a la comprensión de ese momento bistórico. No hay - a priori - razones para saponer la conservación a ultranza de caracteristicas culturales en supuestos desplazamientos, en los que se atraviesan medios distintos, a lo lango de miles de kilómetros y durante miles de años.

El difusionismo tampoco aclaró los mecanismos culturales que hacen posible la difusión de tin rasgo cultural de un pueblo a otro. Esta claro que, para ello, no basta el contacto ocasional entre dos culturas. Deben ast excluirse, como origen de profundas transformaciones culturales, a los probables contactos accidentales que, acaso, se produjeron, por derivas o naufragios, a lo largo de la Prehistoria Americana. Como dato hístórico senálemos que la presencia de los Vikingos en Groenlandia y el Labrador durante cerca de cuatro siglos no "difundió" ningún elemento que pueda ser reconocido entre los indigenas americanos.

No es imposible que alguno de los innumerables rasgos culturales americanos haya sido copiado o adaptado de su similar del Viejo Mundo. Este acontecimiento tiene que haberse producido únicamente cuando dicbo rasgo encajara naturalmente en el estadio de evolución en que se encontraba la cultura americana en cuestión.

Por altimo los progresos de la arqueologia americana han ido produciendo largas secuencias prehistóricas (de hasta 10.000 años en un mismo sítio), que muestran cómo el hombre, desde la ocupación primitiva de los mismos, fue gradualmente modificando sus estrategias de supervivencia, al introducir innovaciones técnicas y desarrolar su estructura social hasta generar las "altas culturas" que encontrarin los europeos en el siglo XVI.

Mientras en el Rfo de la Plata, influido culturalmente por Europa, el difusionismo se imponía, en Norteamérica, fundamentalmente en Estados Unidos, el paradigma dominante era el "evollucionismo", en 
parte de inspiración neodarwinista, La polémica àuí se planteó en torno a cuál era el punto de partida, cronológico y cultural, de las culturas locales.

De este modo los norteamericanos - espiritus pragmáticos y poco dados a las especulaciones - concentraron sus esfuerzos en la investigación arqueológica de las diferentes culturas locales y en su reconstrución prehistórica. Establecieron así las secuencias mencionadas sin gastar sus energias intelectuales en definir probables vinculaciones entre conjuntos de rasgos tomados en forma arbitraria.

Durante las tres primeras décadas del siglo predominó la posición del antropólogo fisico A. Hrdilicka (Hrdicka y otros 1912). En 1911 se encargó de demoler la teoría de un origen americano de la especie humana propuesta por el argentino F, Ameghino (Ameghino 1906), Hrdlicka consideraba a las poblaciones indigenas americanas como racialmente bomogéneas y frato de una inmigración recente, por la vía del estrecho de Bering. Tal inmigración se habria producido a comienzos del Holoceno, hace 9 a 10 mil años. Estos inmigrantes babrian traído consigo un bagaje cultural mesolitico entendiendo por este una etapa de transición entre el paleolítico superior, (donde el bombre vivia principalmente de la caza) y el neotítico (la fuente de recursos principal es la agricultura), Por ser considerado ese momento como el más antiguo se denominó "arcaico".

En 1927 se produjeron los descubrimientos arqueodógicos del sitio de Folsom en Nuevo México (Roberts 1940). Allí se enconiraron, en una asociación indiscutible, puntas de proyectil de piedra tallada y restos de una especie de bisonte que habia vivido en el pleistoceno. A partir de entonces se amplí́ el marco cronológico aceptándose an poblamiento inicial de fines del pleistoceno por parte de grupos de cazadores de la llamada "megafauna" pleistocénica, Se vincularian con sus similares del paleolítico superior del viejo mundo.

Este nuevo marco de referencia suponia una llegada del hombre al continente americano desde hace unos 15 a 20 mil años. Dicha cronologla fue poco a poco precisándose, gracias a la introducción en 1950 de los fechados por $\mathrm{Cl} 4$, en torno a los 11 a 12 mil años antes del presente (el presente arqueológico corresponde al año 1950).

La acumulación de datos arqueológicos hará surgir en la década del 60 una nueva postura (Kricger 1964), de carácter polémico, centrada en 
La existencia de un "hombre temprano". Sostiene un poblamiento primitivo de América muy anterior al de los cazadores de grandes presas armados con puntas de proyectil de piedra tallada.

Con el paulatino abandono del difusionismo en el resto del continente, este nuevo paradigma se transformarí, hasta el dia de hoy, en el centro de las discusiones en torno al poblamiento de América a nivel mundial, dividiendo a los investigadores entre los purtidarios de un poblamicnto "reciente" (de hace 12 mil años) y los partidarios de un poblamiento "temprano" (los más radicales lo remontan a centenares de miles de ańos).

De este modo, principalmente a influjo de la escuela notteamericana, la discusión se ba vuelto principalmente arqueológica y las posiciones se confrontan en función de los resultados de diferentes hallazgos y excavaciones a lo largo del doble continente.

Los antropólogos físicos y los linguístas no son ajenos a esta polémica. En estas áreas se han acumulado investigaciones que permiten uxa mejor comprensión del problema.

De fundamental importancia han sido los progresos metodológeicos desarrollados por ambas disciptinas. Ellos han permitido acortar los limites de las mismas. No tenemos espacio ahora para desarrollar las distintas posiciones a que ban llegado diferentes investigadores; señalemos sí, que no existe aún acuerdo entre los mismos sobre el grado de homogeneidad o diversidad de las razas y lenguas americanas.

Parece, sin embargo, estar formándose un consenso en torno al concepto de que las variaciones linguisticas y raciales son algo menores de lo sostenido en otras épocas y - aspecto trascendente para la comprensión del problema que nos ocupa - , que no existirfa todavia la posibilidad de establecer si esa variación, tanto en un campo como en al otro, es consecuencia de una ewolución local o de una importación por nuevas "oleadas" de inmigrantes;

Los límites de la "glotocronologia" parecen ser insuperables y no son de esperar progresos por ese lado. Por el de la antropologia física podemes aguardar que el avance en los conocimientos sobre la genética, así como la recuperación de células fósiles, permitan algún día establecer un "reloj biológico" que dé una referencia temporal para el momento de la separacion entre los hombres americanos y sus congéneres asiáticos (Greenberg y otros 1986). 
La situacion actual del problema puede restumirse en dos posiciones principales.

La primera, que agrupa a la mayoria de los investigadores, considera que los primeros hombres que llegaron a América to hicieron por la vía del Estrecho de Bering hace aproximadamente $12 \mathrm{mil}$ años. Estos hombres serian portadores de una cultura similar a la del Paleolítico Superior del Vicjo Mundo a la que mayoritariameate denominan "paleoindio". Dicha población primitiva habria luego evolucionado localmente hasta la llegada del europeo. No se descarta la llegada de otras corrientes migratorias, pero estas sólo habrían afectado la parte norte do Norteamérica.

La segunda, on un mayor nêmero de partidarios en Sudamérica, es menos precisa y se centra en la existencia de un problamiento anterior a 12 mil años. No hay consenso en cuanto a la antiquedad del mismo y por to tanto tampoce en cuanto a sus características físicas y culturales. La investigadora brasileña Maria Conccip̧̧ão de Beltrāo, por ejemplo (Beltrâo y Danon 1987), ha defendido la existencia de yacimientos arqueológícos de más de 300 mil años de antiguedad. El poblamiento sería, entonces, obra de pre-sapiens con una cultura del palcolítico inferior. Sin irse a tales extremos, los defensores de un poblamiento "temprano" coinciden en que este fue obra de un muy pequeńo número de pobladores, que portarín una tradición cultural muy primitiva. Estaria caracterizada por la presencia de lascas filosas sin una forma predefinida. No hay consenso sobre el hecho de si las posteriores culturas americanas habrian evolucionado a partir de estos primitivos habitantes, o serían la consecuencia - retomado el difusionismo - de posteriores "oleadas" de pobladores. La bibliografia referente a esta postura es muy extensa, senalemos en particular a Krieger (1964), Brayan (1978), Schobinger (1988), Dillchay (1988), Guidon y Delibrias (1985) y Ardila y Politis (1989) para una descripción de esta.

Para sustentar la hipótesis desarrollada eo este trabajo, sólo tomaremos en cuenta aquellos yacimientos americanos con una antiguedad no superior a los doce mil años, pués como veremos más adelante, los de mayor antigücdad son objeto de diversos cuestionamientos.

Esta exclusión se basa en dos razones fundamentales. La primera, radiea en que los sitios con uns antigüedad superior a dicha fecha no son 
sitios probados (como veremos en el capitulo correspondiente) pues no cuentan con una aceptación mayoritaria de la comunidad cientifica. Toda demonstración debe hacerse en base a hechos irrefutables (probados) y no probables، La segunda razón está basada en el sentido comùn. Este nos indica que no puede haber poblamiento de América por Beringia anterior a la invención de una tecnologia que permita sobrevivir por años en las regiones miśs inhóspitas del planeta, con $0^{n}$ centigrado de isoterma en el mes de enero, por encima de los $60^{\circ}$ de latitud Norte.

Esta tecnología es, sin duda, la del Paleolitico Superior y sus antecedentes más remotos tienen a lo sumo cuarenta mil años en el centro de Europa y tan sólo 15 mil en Siberia Oriental (McBurney 1971, Chmielewski 1971).

Poblamientos de América anteriores suponen el desplazamiento por mar. Este tambićn debe ser excluido en las zonas frias para épocas remotas. Por su parte, la sofisticada tecnologia, capaz de ascgurarle la supervivencia a los pueblos litoraleños del extremo norte, al procurarles las calorias imprescindibles para sobrevivir, está arqueologicamente fechada en el Holeceno (Dumond 1987). En este sentido no sirven las comparaciones con Australia (Hallam 1977) con un poblamiento que parece remontarse a 50 o 60 mil años (Roberts y otros 1990) y que debic atravesar por to menos algunas decenas de kilometros de mares tropicales, partiendo de territorios que ya estaban poblados desde el Paleolitico Interior.

La ocupación de un mundo vacío de hombres debió estar sujeta a determinadas reglas que explican sus características. Las dificultades para reconstituirlas son enormes, pues responden a un tipo de situación sobre la cual los documentos resultan escasos y limitados,

Esta situación clave es la de "frontera".

Debemos entender las características del comportamiento humano cuando enfreata un mundo vacio, abierto, reniendo tras si otro ocupado, donde se compite por los recursos, pero donde también están los parientes, la seguridad del grupo, el socorro ante los imprevistos.

Esta es la gran diferencia con las interpretaciones anteriores relativas al poblamiento $y$ al comportamiento de los grupos que los llevaron a cabo, pues todas ellas ban buscado paradigmas en los testimonios etnográficos e ctnohistóricos tomados en pucblos con un nivel de desarrollo y un conjunto de actividades de subsistencia similares a los 
de los grupes que arqueológicamente se han reconocido como protagonistas del poblamiento americano. Pero estos ejemplos no reflejan la situación de "fronteras", se refieren a poblaciones instaladas en determinado territorio, rodeadas por otras poblaciones que defienden sus propios dominios, Estas condiciones son totalmente distintas a las de la "frontera" donde las posibilidades de expansión no están sujetas a complejas elaboraciones ideofógico-politico-militares.

A partir de datos limitados - pues salvo los arqueológicas habrán sido tomados de un mundo ya totalmente poblado, sacados de fronteras "relativas" donde las poblaciones colonizadoras no se enfrentan a territorios vacios sino escasamente poblados - deberemos reconstruir este tipo de comportamiento.

Las primeras reglas deberán deducirse de la biologia, pues resultan del comportamiento de los hombres como especie, y no ayudarán a la hora de reconstruir el comportamiento social. Es el caso de la capacidad de reprodución, de las limitaciones de la endogamia, de los requerimientos calóricos en distintos tipos de clima, etc (Dumond 1975, Ammerman 1975, Speth 1987).

Un segundo conjunto de reglas se refiere al comportamiento social de estos grupos fronterizos, a su nümero, a su grado de cohesión, su capacidad de innovaciôn, conservadurismo, etc, (Wobst 1976). Ambos conjustos constituyen el gran capitulo de la demografta prehistórica.

Finalmente, en el capitulo denominado el poblamiento de América, pasaremos revista a los principales datos arqueológicos relativos al poblamiento de América produciendo una interpretación coherente de los mismos.

\section{LA DEMOGRAFIA PREHISTORICA}

La reconstrucción del comportamiento demográfico de las poblaciones prehistóricas ante una frontera despoblada es uno de los principios fundamentales para entender el poblamiento do América-

Para ello se debe establecer cuales son las tasas de reproducción y los grados de dispersión posibles. Estas están en relación directa con la potencialidad del territorio, estimada de acuerdo a las necesidades 
calóricas, las que a su wez son dependientes del tipo de actividad desarrollada para procurarse la subsistencia.

Sobre estas bases, que dependen logicamente del modo de vida adoptado, se podria estimar la densidad de habitantes por kilómetro cuadrado.

Es esta dependencia con un determinado modo de vida la que marça los tímites de la expansión inicial de la especie bumana.

Las primeras regiones en set conquistadas por el hombre serán aquellas para las cuales está especialmente adaptado, fisica y culturalmente. Las otras, en primero lugar, las regiones templadas como luego lo serín las frias, representan una verdadera frontera inviolable pues los hombres no están capacitados para vivir en cllas, Dentro de esta frontera y en función del éxito de la adaptación a dicho medio, el género crecerá en número, lenta pero firmemente. Se generan así las condiciones que propiciarán el aprovechamicnto del salto evolutivo que permitirá acrecentar el territorio disponible.

Los primeros hominidos surgen en Africa hace aproximadamente dos millones de años. Están purticularmente adaptados a un clima cálido en un medio caracterizado por la frontera entre el bosque y la sabana (White 1982, Taieb \& Poupeau 1980). Su acervo cultural, caracterizado por guijarros tallados y algunas lascas simples, y su capacidad biológica los limitan a este medio al que ocuparán durante un millón y medio de años.

Mucho se discute sobre cuál habrá sido el modo de vida de los Homo Habilis, tratando de estimar el peso relativo de la dieta de origen animal o vegetal. Parece existir un concenso sobre el becho de que si bien la carne un elemento fundamental en la dieta, esta no babria sido obtenida mediante técnicas de caza "ofensivas", sino de una manera "oportunista", aprovechando animales muertos por los graades carnivoros, o ultimando aquellos que se encontraran en un grado de debilidad extrema, a la manera de las hienas (Shipman 1986, Lee 1981, Klein 1977). Este modo de subsistencia. "carron̄ero" con su dependencia estrecha hacia medios ecológicos restringidos es el que babría limitado la expansión de esta especie.

Hace aproximadamente un millon de años se habria producido el primer salto evolutivo, tanto biológico como cultural. Aparece el llamado Homo Erectus, con una mayor capacidad craneana y una cultura, 
sofisticada en relación a la anterior, caracterizada por la presencia de bifaces y lascas retocadas.

Este equipo técnico más perfeccionado, permitirá romper la estrecha dependencia, con el medio ecológico del período anterior. El Homo Erectus ocupará entonces la totalidad de las zonas cálidas del planeta y hará una primera incursión en las áreas templadas.

No es fácil determinar - tampoco para los Homo Erectus - cual fue su modo de subsistencia. Algunos yacimientos situados cronológicamente cerca del final del periodo, como los de Torralba y Ambrona (Howell \& Freeman 1982), estarian indicando la existencia de un tipo de caza más ofensivo, atacando con palos de punta endurecida por el fucgo e animales que se encontraban en condiciones de inferioridad.

Es el momento previo al inicio de las glaciaciones cuaternarias cuando el clima cra más cálido que el actual y los elefantes llegaban hasta las Islas Británicas.

Con el inicio de las glaciaciones, hace aproximadamente unos seiscientos cincuenta mil años, la especie humana será sometida a un considerable estres por las modificaciones en el efima y las consecuentes variaciones en los territorios ocupables.

De este modo se generarín nuevamente las condiciones en que la "supervivencia del más apto" será definitoria. Los más aptos, hablando de hombres, son aquellos que pueden modificar su cultura de acuerdo a las nuevas situaciones a enfrentar y estos serán a su vez los que dispongan de una base biológica mejor adaptada para cstos fincs. Surgen ahora los primeros "Homo sapiens" y un nucvo desarrollo de las culturas paleoliticas, conocido como "Paleolítico Medio".

Este período que comienza hace unos doscientos mil años permitirá terminar la colonización de las zonas templadias del plancta e iniciar el de las zonas frias aledañas.

Es necesario aclarar que, hasta el momento, no hay prucbas fehacientes de la presencia del hombre en las zonas frias, a más de 55 grados de latitud norte, antes del Palcolítico Superior. Se ha señalado que algunos sitios arqueológicos correspondientes al paleolítico modio y aún inferior se encontrarian dentro de áreas de clima frío, pero salvo para algunos de Europa Central que cultural y cronológicamente se encuentran en el periodo de transición con el paleolítico superior, los datos no son 
claros pues se encueatran mezcladas faunas de periodo cálides y fríos (James 1989, Bordes \& Thibault 1976).

Recordemos que nos encontramos en las últimas etapas del periodo glaciar y que durante las glaciaciones buena parte de los territorios hoy emergidos se encuentran bajo espesas capas de hielo. Las regiones nórdicas que no lo están, se caracterizan por la presencia de tundras y estepas heladas (Watanabe 1971). Esto tiene implicancias demográticas y geográficas.

La acumulación de agua en los glaciares provocó un descenso de decenas de metros en el nivel de los mares haciendo emerger vastas zonas costeras que hoy se encuentran sumergidas. De este modo el territorio que se perdía por un lado era en cierta medida recuperado a lo largo de las costas, Asi se produjo también la emersión del territorio del estrecho de Bering, que unia en un sólo continente Asia y Amćrica, formando un puente ferrestre de basta mil ochocientos kilómetros de ancho por el que pudieron intercambiarse plantas $y$ animales (Hopkins y otros 1982).

Fibalmente, hace aproximadamente cuarenta mil atios, se produce el último salto tecnológico evolutivo con la aparción de nuestra propia especie, el Homo Supiens Sapiens, y de un nuevo desarrollo eultural conocido como "Paleolítico Superior".

Será el hombre del Paleolítico Superior el encargado de conquistar las zonas frias del planeta y quien, en sus peripecias a través de éstas, llegará a América colonizando la llamada "Beringia" que en aquel momento unía, formando un todo, Siberia Oriental y Alaska.

Los hombres del Paleolítico Superior llevarín a su máximo grado de perfeccionamiento la talla de las rocas duras, produciendo finas lascas conecidas como láminas y también puntas de proyectil, adelgazadas bifacialmente mediante la técuica de la presión. El prototipo mejor conocido son las llamadas "hojas de lauret" de altas performances balisticas.

Estas puntas de proyectil bifaciales en piedra tallada y la aparición de ctras en hueso pulido, constituyen um acervo técnico, que junto a los numerosos restos de animales muertos, y los testimonios del arte parietal y mueble, nos están indicando claramente una cconomía basada en la caza ofensiva de grandes herblvoros. Aclaremos que el caracter fundamental de la caza de grandes animales está probado arqueológicamente por la preponderancia en la elaboración de instrumentos para esse fin, y en la 
distribución desigual de los restos de fauna en los vacimientos. Esto por supuesto no excluye el aprovechamiento de otros recursos, vegetales o litoraleños, que complementaban la dieta y que en algunas regiones (que no son precísamente las zonas heladas para el caso de los vegetales) pueden haber sido los principales.

Este tipo de caza mayor supone una organización del trabajo que tiene implicancias de tipo social, que no podemos trasladar con certeza para los períodos anteriores. El enfrentar los rebaños de grandes herb́fvoros, como el mamut por ejemplo, necesita de verdaderas estrategias de caza en las que debian participar un número minimo de individuos organizados y dirigidos. Asimismo, el aprowechamiento de estas grandes presas que nos podían ser consumidas en forma inmediata, supone criterios de almacenamiento y distribución entre aquellos que no han participado directamente en la caceria.

Estas implicancias nos están hablando de una mayor complejidad social que repercute directamete en la interpretací́n de nuestro tema, pues de ella dependerá también, junto con las determinantes biológicas, el número mínimo de individuos que deben componer un grupo de cazadores de grasdes presas del Paleolitico Superior.

El éxito de esta economía queda demonstrado tanto por aumento en el número de sitios como por la propia colonización de las estepas frías, donde pastaban rebaños de animales adaptados al clima y que haste ese momento no habian sido aprovechados.

Es este lento peregrinar del género humano, pautado por los progresivos saltos en la evolución biologica y cultural que le posibilitan afrontar zonas cada vez más iñóspitas, al que denominamos "paradigma universal", el que nos permite comprender el poblamiento de América como el último paso del mismo.

Debemos aclarar que no se propone aquí que dichos saltos evolutivos se hicieran en función de la necesidad de colonizar las zonas deshabitadas para aliviar la presión demográfica. Sin excluir esta posibilidad, pensamos que lo mas probable es que los mismos se hayan producido al interior de las fronteras donde esa presión tendria mayores probabilidades de manifestarse. Esos saltos tecnológicos que permitían un mejor aprovechamiento de las áreas ya połladas generarán a su wez la potencialidad de utilizarlos en la conquista de nuevo territorios: 
Señalemos un concepto fundamental para esta interpretación: desde el punto de vista estrictamente técnico no hay ninguna "barrera" aingún "salto" desde que los primeros Homo habilis golpearon dos guijarros de rocas duras - de fractura concoidal - para obtener un borde filoso, hasta que los Homo sapiens sapiens del paleolitice superior elaboraron las sofisticadas puntas "hoja de laurel".

Las distintas etapas tecnologicas del paleolitico ban consistido, en términos generales, ûnicamente en agregar nuewos "golpes" a los iniciados por el Homo habilis. De este modo en lugar de tener un "Chopper" (guijarro sobre el cual se ha lagrado un borde cortante retirando unas pocas lascas), agregando más golpes y retirando más lascas sobre ambas caras, obtendremos un "bifaz". Agregando más golpes al bifaz conseguiremos reducirlo a una fina hoja que puede ser utilizada como punta de proyectil.

Por supuesto que hubieron variantes $e$ innovaciones: percutores "duros" (piedra) o "blandas" (bueso, cuerno, madera), golpes "directos" e "indirectos", con el núcleo de materia prima apoyado o suelto, o finalmente ejerciendo presión en lugar de golpear. Es indudable que el principio general es el mismo y que quien puede fabricar un Chopper, si es capaz de concebirla 0 , si realmente la necesita para sobrevivir, pucde también fabricar una "Hoja de laurel".

Este es un aspecto importante a la hora de la interpretación arqueológica de los hallazgos paleoliticos y debe ser tenido en cuenta para explicar la falta de correlación tipológica entre los conjuntos paleoliticos de las áreas cálidas y de las áreas frias y templadas. Esto serí tetomado a ta hora de interpretar los hallazgos americanos.

Se ha calculado la población a fines del Paleolitico entre 3 y 5 millones de habitantes lo que, tomando como punto de partida dos millones o un millón de años, daria una tasa de crecimiento demográfico del 0.0007 al $0.0015 \%$ por año (Dumond 1975:717), La superficie terrestre habitable comprende 136 millones de kilómetros cuadrados y la densidad de población promediaría los 0.03 habitantes por kilómetros cuadrado. Esta densidad debe ser comparada con la de las poblaciones cazadoras recolectoras conocidas etnográficamente, que ha estimada en un máximo de 0.4 habitantes por kilómetro cuadrado.

No conecemos estimaciones sobre la población prehistórica en el paleolitico inferior y medio, estimación harto difícil dada la escasez de 
restos humanos. Parece bastante evidente, que (aún teniendo en cuenta la pérdida de sitios por el mayor ticmpo transcurrido) los sitios arqueológicos fueron en aumento a lo largo de todo el Paleolítico y que cl crecimiento de población resulta en términos generales permanente (Cohen 1981).

Existen estimaciones sobre la esperanza de vida de las poblaciones presapiens así como algunas especulaciones sobre sus tasas de reproducción en función de esta y también por comparación con nuestros primos los chimpancé. Estats especulaciones son de dificil manejo y debemos asignarles una importancia relativa. El becho es que, posiblemente, con tasas reproductivas extremadamente conservadoras estas primeras especies de hombres lograron ocupar y poblar la totalidad del medio que estaba técnica y biológicamente a su alcance.

Partamos entoaces de la situación a comicnzos del Paleolitico Superior con nuestra propia especic, euyo comportamiento podemos estimar en forma más fiel, en visperas de lanzarse a la conquista del territorio aún desocupado del planeta, la frontera, con los aproximadimente 20 millones de kílometros cuadrados de tundras y estepas de Europa, Siberia, Beringia, Alaska y Canadá.

Los estudios sobre cazadores recolectores históricos ban mostrado cómo estos se desplazan sobre un territorio detimitado.

Según L. Binford, en el caso de los esquimales Nunamiut, el territorio está divididoengrandes áreas, cada una de las cuales sirve como base para los desplazamientos estacionales. Estos se repiten durante algunos atios hasta que el área comienza a ser deficitaria y entonces se desplazan a otra de las áreas de su territorio donde recomienza el circuito estacional. El recorrido total del territorio del grupo puede llegar a compreader una generación. Al mismo tiempo, como grupo, permanentemeate tienen conciencia de la totalidad del territorio que ellos controlan, asi como de la potencialidad de sus recursos (Binford 1988:220-4).

Este ejemplo de poblaciones adaptadas al ártico puede servirnos de base a la hora de interpretar los primeros desplazamientos por la zona helada.

El grupo humano situado en la frontera de las regiones frias conece y controla un determinado territorio, En la medida que desarrolla una tecnologia que ke permite aventurarse en dichas zonas empezará a incluir como área de posible aprowechamicnto las cercenas a so punto de partida, 
sin abandonar a priori este, pues conoce su potencialidad que se renuevat periódicamente. La causa probable del abandono del antiguo territorio por parte del grupo, parece ser, razonablemente, el crecimiento demográfico.

Estas nuevas tecnologias, más eficaces hacia el interior del nuevo territorio, desarrollan la capacidad depredadora de la especie al mismo tiempo que estimulan - por mayor disponibilidad de recursos - el crecimiento demográfico, acelarando la llamada "presión de población" y estimulando la necesidad de nuevos territorios.

Coincidentemente, la disposición "ilimitada" de recursos por efecto de una frontera abierta, tiene que haber incidido decisivamente en el comportamiento reproductor. Este se relaciona bistóricamente con la conciencia que el grupo tiene sobre la disponibilidad de bienes (Dumond 1975: 719-20).

1.Que frena entonces el desarrollo de una verdadera explosión demográfica y la rápida colonización del área belada?

La respuesta se encuentra seguramente en el medio inhóspito sobre el que se desarrolla la colonización (ver infra p. 13 y 19). Esta contaba con una tecnologia cazadora eficaz para proveer las 3.800 calorias diarias necesarias para sobrevivir en el medio helado, pero que está en el límite de sas posibilidades. No olvidemos que la demografía es el cociente entre los nacimientos y las muertes y estas pueden haber sido significativas en un medio de extrema dureza.

\section{EL POBLAMIENTO DE SIBERIA}

De acuerdo a lo expuesto el poblamiento de América sólo pudo haberse producido una vez logrado el poblamiento de Síberia.

El territorio siberiano comprende una enorme superficie (unos 10 millones de kilometros cuadrados) y su conocimiento arqueológico se ve. dificultado por la baja densidad de población y las dificuldades que el propio medio geográfico plantea. De este modo debemos tener presente que los datos que manejamos (enrarecidos a su vez por las dificuldades de acesso a materiales en lengua rusa, excepcionalmente traducidos al inglés) son de carácter primario (Michael 1984:1-2). El bosquejo de la prehistoria 
siberiana que de ellos se deduce seguramente resultar alterado por nuevas investigatciones.

El registro arqueológico disponible (Okladnikov \& Pospelova 1982, Klein 1971, Micbael 1964 y 1984, Dikov \& Titov 1984, Okladnikov 1961 . Mochanov 1978, Tolstoy 1958) nos muestra que los sitios más antiguos se encuentran al occidente de Siberia, en la llanura aluvial del río Ob y se van haciendo cada vez más recientes, cuenca del Yenissei, lago Baikal, ríos Lena y Aldán y finalmente peninsula de Kamchatka. En ningùn caso están comprobados asentamientos anteriores al ultimo episodio glacial ( 30.000 10,000 A.P.) denominado Sartín para Siberia (KJein 1971:136-138).

Debemos entonces, en principio, descartar que la ocupación de Siberia se hubiera producido mediante desplazamiento de poblaciones en sentido Sur-Norte. Esta ausencia de pruebas arqueologicas es coherente con las dificuldades de indole geográfica que plantearia esta ruta de migración, como la presencia de las grandes cadenas montañosas de Mongolia, con la consecuente formación de glaciares y, principalmente, por la presencia de un medio sumamente inhóspito, de una verdadera frontera natural, el bosque de coniferas - la taiga - que se extiende sobre el territorio sur de Siberia, prolongindose hasta el Océano Pacífico (Klein 1971:143, Vaskovskiy 1964:494-496), El besque de coníferas supuso una menor disposición de recursos para los cazadores del Paleolitico Superior en relación a las estepas frías y la tundra, donde se encontraban las manadas de grandes herviboros, su fuente principal de alimentos. De este modo, pese a corresponder a un medio menos frio, era toológicamente más dificil de aprocechar y su ocupación será entonces más tardía, una vez agotada la posibilidad de colonización de las zonas esteparias (Watanabe 1971).

La adaptación al medio frio parece heberse producido entonces a partir de las poblaciones paleolíticas del este de Europa, como lo estarían indicando los sitios de Rusia, Ucrania y Bielorusia fechados desde 36000 a 33000 A.P. (McBurney 1971:238, Dmitriyeva 1980: $828-829$ ver también Budko 1971, Schovkoplyas 1971), las que cruzando los Uraies se habrian extendido hacia el este. Sin embargo debemos tener presente, que en base a los datos actuales, no podemos descartar totalmente que dicha adaptaciôn se haya producido también, aunque más tardímente, en otras regiones fronterizas con Siberia, como el Asia Central y Manchuria, y que desde allí se haya extendido hacia el Norte y el Noreste. 
El tema del origen de las primeras poblaciones siberianas es clave desde el punto de vista de la antropologia biologica americana pues el mismo está relacionado con el discutido problema del origen del bomo sapiens sapiens y de las diferentes razas humanas inclayeado el supuesto carácter "protomongolóide" de las poblaciones americanas.

Lamentablemente disponemos de aûn menos información sobre la arqueologia de la ex Asia Central soviética donde estarian registrados yacimientos correspondientes al paleolitico superior que habrian sido precedidos por una muy probable ocupación "musteriensc".

Veamos entonces los datos correspondientes a la Prehistoria Siberiana, ordenándolos en primer lugar en funcí́n del registro cronológico (elemento clave para determinar la fecha del probable pasaje al territorio americano) para luego considerar las tradiciones culturais (también deben ser relacionadas con las que se encuentren en América).

Con el mismo criterio que aplicaremos a los yacimientos americanos, elaboremos esta síntesis únicamente en función de los sitios cronológicos aceptados, dejando expresamente de lado aquelles cuya asignación es objeto de polémica.

De este modo se dibuja un cuadro bastante coherente en el cual los sitios más antiguos se encuentran en el Oeste y se van haciendo cada vez. mảs recientes a medida que avanzamos hacia el Este. Repitamos que no debemos perder de vista que estamos tratando con algunas decenas de sitios desparramados sobre un territorio de millones de kilómetros cuadrados.

No hay en Siberia - con la unica c improbable excepción del sitio de Ulalinka (Okladnikov \& Pospelova 1982:710-712) - yacimientos correspondientes al paleolitico inferior $y$ medio y los sitios que men. cionaremos corresponden todos, cronológics y culturalmente al $\mathrm{Pa}$ feolitico Superior.

Los sitios mấs antiguos se encuentras al oeste del lago Baikal: Malta, en la evenca superior del Angara y Afontova Gora, en el curso medio del Yenissci (aproximadamente $100^{\circ}$ E y $50^{\circ} \mathrm{N}$ ), que están fechados en sus niveles más antiguos en 23000 y 21500 A.P. respectivamente. Luego 5eguirian los fechados que corresponden a los sitios del trans-Baikal y del valle del río Aldán $\left(60-63^{\circ} \mathrm{N}, 130-135^{\circ} \mathrm{E}\right)$, dende destacaremos los de la cueva Diuktai (13400 A.P.) y el sitio de Bareleck, el más nórdico que se haya registrado $\left(71^{\circ} \mathrm{N} 140^{\circ} \mathrm{E}\right)$, con $12800 \mathrm{~A}$. P. Luego el sitio de Ushki en 
La peninsula de Kamtchatka $\left(60^{\circ} \mathrm{N}, 160^{\circ} \mathrm{E}\right)$ - el más próximo it América - con 14300 A.P. Finalmente los sitios del curso-superior del rio Kolyma, zona obligada de pasaje para un desplazamiento sur-norte a lo largo de la costa del mar de Okhotsk, con sus fechados notablemeate tardíos de 10000 A.P. (datos de Michael 1984, Dikov \& Titov y Klein 1971).

Este cuadro es cuestionado ûnicamente por los fechados de los sitios sobre el río Aldán, en particular el de Ezhantzy, propuestos por el investigador Y. Mochanov (1978), las que harian retroceder la ocupación de Siberia - aunque siempre dentro de los límites cronológicos y culturales del Paleolítico Superior - a 35000 años A.P. Estos fechados ban sido cuestionados (Dikoy \& Titov 1984-69-702, Hoffecker 1989:417) y son cuestionables por la incoherencia entre las fechas $y$ las características tipológicas que habrian permanecido inalteradas durante $\mathbf{2 1}$ mil años (Michael 1984:16). Hemos retenido aqui los fechados de la cueva de Diuktai, que da el nombre a la cultura epónima, por la seguridad que nos brinda su estratigrafia.

El prehistoriador debe tener presente que la reconstrucción paleogeográfica de Siberia Oriental es particulármente compleja, pues la zona se encuentra cortada por númerosas cadenas montañosas, desde las caales se extendieron calotas glaciales, influyendo en la extensión de los sistemas de tundra y de taiga (Vaskovskiy 1964). La geografia present6́ pues, serias dificuldades para el avance de las poblaciones humanas. No debemos imaginarnos una extensión geográfica uniforme, donde los hombres hubieran podido expandirse libremente y a un ritmo constante, sólo determinado por las características demográfico-culturales de dichos grupos.

Desde el punto de vista de su acervo cultural los sitios siberianos pueden dividirse en dos grandes tradiciones, ambas tipológieameate adscriptas al Paleolitico Superior.

Por un lado los sitios bien conocidos del cis-Baikal de Malta y Buret $\left(53^{\circ} \mathrm{N}, 103^{\circ} \mathrm{E}\right)$ donde se desarrollo una tradición de tipo "auriáaciense" con enfasis en la fabricación de láminas y el trabajo del hueso y del marfil, pero sin presencia de puntas de proyectil bifaciales en piedra tallada (de tipo "foliáceo" o "leptolitico") (Gerasimov 1964). Por el otro, la tradición correspondiente a la llamada cultura Diuktai. Se identifica por la fabricación de puntas de proyectil foliáceas y de laminillas o micro láminas, extraidas a partit de núcleos especialmente preparados, cono- 
cidos bajo el nombre de núcleos "en cuña" o nû̀cleos "gobi" (Mochanov 1978).

Ambas tradiciones culturales parecen haberse baseado en la caza de los herbívoros de las estepas, particularmente del Mamut, para ta sat lisfacción de sus necesidades principales.

Los tipos de asentamiento, cuando han podido ser reconocidos como en el caso de Ushki, corresponden para sus primeros niveles a grandes vivicadas de $100 \mathrm{~m}^{2}$, construidas en parte con huesos de mamuts, que deben de haber servido de residencias colectivas (Dikov \& Titov 1984:74).

Poco podemos adelantar aquí sobre la reconstrucción de su modo de vida y sobre una explicación de las diferencias entre estas dos tradiciones culturales. Estas se encuentran separadas en el tiempo y en ef espacio por distancias lo suficientemente apreciables como para generar diferencias en el registro tipológico. Inversamente, sería la conservación de rasgos similares lo que debería llamarnos la atención, pues no ocurre en ninguno de los regístros arqueológicos conocidos para el paleolítico superior, ni en una misma región, y menos aún, sobre decenas de miles de kilómetros cuadrados (un área comparablt a la superficie de Europà).

\section{EL POBLAMIENTO DE ALASKA}

En Alaska se repiten las mismas dificuldades que en Siberia para establecer de un cuadro arqueológico relativamente completo.

Sin embargo, pese a estas, ba sido el escenario de una búsqueda sistemática de sitios que pudieran aportar respuestas al problema del poblamiento (Hoffecker 1988, Dixon 1976 y 1985, Ten Brink y Waythomas 1985).

Estas investigaciones han resultado pobres en materia de hallazgos, pero muy ricas en información geomorfológiea y paleoecológica. Estas nos permiten conocer los límítes de las prospecciones arqueológicas y las dificuldades que el medio opuso al avance de los primeros pobladores. Dos bechos parecen ir conformándose. Por un lado, los sedimentos correspondientes al cuaternario han sufrido profundas alteraciones y los contextos geomorfológicos donde podrían encontrarse vestigios de un poblamicnto anterior a 13 mil años son relativamente escasos. Limitan 
entonces las posibilidades de encontrar sitios anteriores a esta fecba. Por el otro, en aquellos lugares donde estos suelos existen, las prospecciones no han arrojado resultados positivos sobre la presencia humana más allá de 12 mil años A.P. y tampoco, lo que seria una evidencia indirecta, donde se encuentran redepositados los sedimentos removidos, tampoco bay presencia de herramientas en piedra tallada.

Los sitios más antiguos de Alaska tienen aproximadamente 12 mil años de antiguedad aunque el sitio de Bluefish Cave en el Yukón podría tener hasta 15 mil años (Hoffecker 1988: 109). Los sitios con fechados más antiguos se encuentran en la cuenca del rio Nenana, destacándose en particular Dry Creek y sitios vecinos con fechades de 11800) A.P. (Powers y Hoffecker 1989).

Desde em punto de vista cultural, dado lo reducido de la muestra, la determinación no es definitiva aunque es indudable que estas industrias presentan claros paralelos con el paleolítico Sibcrianc.

Existe un estadio cultural bien definido, conocido como cultura Danali que se caracteriza por el énfasis puesto en la fabricación de laminillas, con claras relaciones tipológicas con la cultura siberiana de Diuktai. Se discute si se puede definir un estadio anterior "pre-Denali" establecido $\mathrm{cn}$ base a una mayor preponderancia de las puntas de proyectil y un menor énfasis en la fabricación de laminillas. Se han propuesto las denominaciones de "complejo Nenana" y "complejo Chindadn" para esta etapa (Dixon 1985, Powers y Hoffecker 1989).

Una estratificación similar se daria en Kamtchatka, en el sitio de Ushki, donde para los primeros niveles las puntas serian el elemento preponderante (Dikov y Titov 1984: 74). Es dificil establecer una situación definitiva puesto que en ambos sitios, los diferentes hallazgos de artefactos se encuentran agrupados por zonas, hallándose concentraciones de laminillas por un lado y concentraciones de puntas bifaciales por otro. Esto puede dar como resultado el sesgamiento de la muestra, si la misma no provienc de excavaciones a gran escala.

No tenemos, para la arqueología de Alaska, reconstrucciones sobre el tipo de habitat que permitan hacer comparaciones con Siberia. Los restos culturales estấn asociados a fauna extinta. Las puntas de proyectil son del tipo lanceolado, triangulares y en forma de lágrima (Dixon 1985, Powers y Hoffecker 1989). Hay que señalar el hallazgo en forma aislada 
de puntas acanaladas similares e las que luego encontraremos hacia el sur del continentes norteamericano.

Estas, en los pocos casos en que han sido hallados en contextos fechables, se ubicarian entre el 11 y el 10 milenio A.P. (Dixon 1976).

\section{EL POBLAMIENTO DE NORTEAMERICA Y SUDAMERICA}

Con el problamiento de Alaska sc cierra el capitulo de la colonización de las estepas beladas del norte da Asia, de "Beringia" $\mathrm{y}$ de América. Superada la barrera planteada por los casquetes glaciales de las Rocosas y del Escudo Canadiense, se abrirá para la especie humana la etapa "verdaderamente americana" del proceso de colonización se invierte: la bumanidad ocupará ahora latitudes cada vez mas benignas, climas templados; sabanas tropicales.

Se genera así un muy interesante problema antropológico: icomo reaccionará culturalmente lut especie? ¿qué sucede con el desarrollo tecnológico cuando falte la presión para un mejoramiento contínuo? ¿qué acontece con los lazos sociales cuando la supervivencia del individuo, antes determinada por una estricta disciplina social, ahora puede asegurarse autónomamente? ¿qué ocurre con el comportamiento demográfico, necesariamente controlado al ser los recursos limitados y ahora $\sin$ trahas?

Esta fué, sin duda, la única humanidad sin fronteras en todos los planos, mucho mas que sus abuelos periárticos, quienes tentan una frontera, despoblada de hombres pero delimitada por el conocimiento del medio (garantfa de subsistencia) y la ignorancia y agresividad del medio a colonizar.

Veamos primero los datos de la arqueologia para analizar luego el problema de la interpretación prehistórica.

Los casquetes glaciales de las Rocosas y de las Laurentidas representaron una barrera para el avance de las poblaciones prehistóricas. Separaban claramente los medios ecologicos a colonizar: la tundra al norte y las grandes praderas al sur.

Durante los interglaciares, cuando las temperaturas extremas se atenuaban ambas masas glaciares se retraian, dejando libre de hielos un 
territorio que ha sido denominado "corredor del Mackenzie". Por el se desplazaron la megafauna pleistocena y el hombre. Esta coincidencia entre la apertura del "corredor" y fa presencia humana al interior de Alaska reciên se habría podido producir en el interestadio de Two Creeks, finalizado hacia el 11400 A.P. (Haynes 1988; 8), y posterior en algunos siglos a los primeros yacimientos arqueológicos de Alaska.

Sen̂alemos que, sin embargo, no estä definitivamente establecido cn cuáles períodos de máximo de frio hubo coalescencia de los glaciales. Tampoco se puede descartar en forma absoluta (aunque requeriria una adaptación a las costas heladas no registrada para esa época) la via de penetración a lo largo de la costa del Pacífico (Fladmark 1979).

Posteriores al interestadio Two Creeks son los primeros yacimientos fechados al sur del continente norteamericano. Estos se exticnden de manera uniforme desde el centro y basta ol este de Norteamérica, concentrándose en los ûltimos tres siglos del décimo segundo milenio. Esta cultura se caracteriza por su uniformidad tipológica, pot su gran dispersión geográfica, por el relativamente breve periodo durante el cual se desarrolló, por la importancia de la megafauna (principalente el mamut) como recurso alimenticio y por la repetición de los patrones de asentamiento cuando estos han podido ser identificados (Kelly y Todd 1988).

Esta uniformidad cronológica y cultural se conoce bajo el nombre de Cultura Clovis, cuyos restos han sido identificados en decenas de sitios en toda Norteaméricá, desde México a Canadá.

Tipológicamente su acervo es característico del Paleolítico Superior. Se considera la primera manifestación cultural de este cstadio en América. Muchos autores, negándose a utilizar una terminologia de valor universal, lo denominan "Paleoindio" (Schobinget 1988b).

Se diagnostica por la presencia de las llamadas puntas Clovis. Dichas puntas - talladas bifacialmente, retocadas a presión y hechas sobre rocas cripto-cristalinas cuidadosamente seleccionadas - se caracterizan por presentar, en su parte proximal, uno o mas negativos de lascado que las adelgazan en la parte central de la base.

Estos negativos - presumiblemente destinados a facilitar el enmangado de la punta y/o, quizás, para aumentar los efectos de la berida producida - Ie dan características enteramente originales, sin paralelos en el Vicjo Mundo, haciendo que se describan como puntas "acanaladas" 0 "aflautadas". 
El conjunto tipoløgico está compuesto asimismo por láminas (sin laminillas) y artefactos realizados sobre estas y sobre lascas, destinados fundamentalmente al trabajo de la carne, el cuero y el hueso y constituido principalmente por bifaces, raspadores, cuchillos y perforadores.

La cultura Clovis contiene un menor número de tipos en relación a tas culturas de Alaska y de Siberia y es reducido en cuando a la diversidad de piezas, estando compuesto por aproximadamente 20 tipos distinos (Irwin y Wormington 1970).

Es de señalar la cuidadosa seleción de la materia prima utilizada, en muchos casos traida desde más de 100 kilómetros de distancia. Esta economia de la materia prima debe necesariamente relacionarse con la fugacidad y movilidad de estos grupos.

Los asentamientos parecen baberse realizado preferentemente al aire libre en lugares estratégicos relacionados con los puntos de agua (no conociéndose yacimientos Clovís localizados en cucvas o abrigos) y están muchas veces relacionados con lugares de matanza, principalmente del mamut (Haynes 1966). Es característica también la escasa concentración de vestigios arqueológicos en los mismos lo que indicaria una ocupación limitada en el tiempo.

Es interesante agregar a este ûltimo aspecto mencionado que junto a algunas decenas de sitios Clovis daramente identificados, debemos agregar el hallazgo de miles de "puntas Clowis", aisladas, en superficie o fuera de contexto, a lo largo y a lo ancho de todo el continente norteamericano y aûn de centroamérica. Indiean el área de dispersión de esta cultura y a su vez, confirmarian la brevedad de la ocupación de los sitios.

Otro elemento a sev̈alar (junto con el anterior podría ser un indicador de una baja densidad demografica) es que en los sitios de matanza no es posible identificar un aprovechamiento exhaustivo de las earcazas. La mayoria de las piezas del esqueleto no han sido desplazadas en forma sítemática ni se ban registrado depósitos organizados de huesos que pudieran indicar una actividad de destazado destinada a la obtención de reservas de alimentos.

Los datos disponibles sobre la distribuición en el tiempo y en el espacio de los sitios Clovis en el norte de América permiten definir este primer episodio de la Prehistoria Americana como un "horizonte" en seatido arqueológico. Es decir la distribuición geográfica extensa de una manifestación cultural bomogénea, durante un periodo relativamente breve. 
Los datos para América Central y América del Sur son, por su parte, mucho menos claros y abundantes, por lo tanto tendremos que reconstruir el primer episodio del poblamiento de Suramérica en forma tentativa, en base a algunos pocos datos aislados (Schobinger 1988, Ardila y Politis 1989). Estos permiten inferir que el "Horizonte Clovis", modificándose desde el punto de vista tipológico a medida que avanza bacia el sur conserva, empero, los rasgos distintivos fundamentales de la dependencia de la caza en su economía y el "aflautado" o "acanalado" en la base de las puntas de proyectil. Alcanzando rápidamente el extremo sur del continente.

Estas primeras puntas sudamericanas se caracterizan por tener el limbo avalado con pedúnculo (las puntas Clovis no lo tienen) y por presentar, en muchos casos, en la base de este los negativos de adelgazamiento que las vinculan con la tradición de las puntas "aflautadas". Han sido denominadas "Cola de Pescado" (Schobinger 1973).

Esta hipótesis es avalada por los escasos sitios sudamericanos donde se ban encontrado puntas Cola de Pescado en contextos con fechados indiscutidos. Son los sitios de: Fell y Palli Aike (Bird 1969) en el extremo sur de la Patagonia Chilena; Cueva Medio, Provincia de Ultima Esperaza, Chile (Nami 1989); Los Toldos, Provincia de Santa Cruz, Argentina (Cardich 1977) y Cerro La China, Provincia de Buenos Aires, Argentina (Flegenheimer 1987). Todos ellos presentan fechados que se ubican entre 10500 y 10750 A.P., siendo los de la Provincia de Buenos Aires los más antiguos.

A estos sitios - que sugeririan uns manifestación regional de estas puntas - debemos agregarles los hallazgos en los sitios estratificados de El Inga en Ecuador, $y$ de Pikimachay (fase Huanta) en Perú, con una cronologia menos segura y multitud de hallazgos en superficie a lo largo y ancho del continente sudamericano (Schobinger 1973).

Todos estos sitios tienen los tres elementos que a nuestro juicio son determinantes de una relación directa con el horizonte Clovis. En primer lugar, su cronología, pues son los sítios más antiguos de Suramérica y sólo posteriores a Clovis en algunos siglos.

En segundo lugar, su tipologia, que sigue conservando las características leptoliticas de las industrias del Paleolitico Superior y que, si bien ha sufrido modificaciones, está estilísticamente relacionada con Clovis. Esta relacion se funda principalmente en las puntas de proyectil, denominadas "cola de pescado" o "Fell $\mathbf{I}$, que conservan el rasgo más 
singular - sin paralelos en el Viejo Mundo - de las puntas Clovis: La acanaladura en la base de las puntas.

Se difereacian de las Clovis por ser pedunculadas y por tener el limbo de forma ovulada. Al parecer habrian ido diferenciándose de las originales a medida que se avanzaba hacia el sur. Es asi que en territorio mexicano se conocen puntas tipicamenté Clovis y en América Central encontramos, en el sitio del Lago Madden en Pansmá, puntas de forma más ovalada, en las que se insinúa un pedúnculo, que podrian ser el nexo entre las típicamente Clovis de Norteamérica y las Cola de Pescado sudamericanas (Bird y Cooke 1978).

En tercer lugar, la dependencia económica de la megafauna pleistocena - caballo, megaterios, paleo-liamas - que repite las pautas de la cultura Clovis en casnto a su dependencia de las grandes manadas de herbivoros.

Tanto en América del Norte como en América del Sar, este primer episodio de ocupación del continente parece haber durado solo unos pocos siglos, durante los cuales la especie humana se expandío rápidamente. Lo refleja asi la uniformidad cultural anteriormente descripta, que ocupa todas aquellas áreas donde el sistema de vida del cazador de megafauna podía ser reproducido (Kelly y Todd 1988).

La finalización de este episodio se evidencia en la regionalización de los registros arqueológicos, en el fin de los "horizontes" que caracrerizaban el poblamiento inicial.

Las tradiciones estilisticas en el tallado de la piedra se harán ahora eada vez más locales, reduciéndose a ăreas delimitadas. Simultaneamente se inician nuevas pautas culturales, relacionadas con la ocupación de medio-ambientes que, al no estar densamente poblados por grandes maniferos, no habian sido ocupados inicialmente.

Estos requerirain otras estrategias adaptativas basadas principalmente en la recolección y/o en la captura de peces y pequeños animales (Hayden 1981).

Lógicamente, el estilo de vida cazador (en el sentido de la preponderancia de la caza de grandes animales) perdurará en aquellos lugares donde las condiciones asi lo permitan. El fenómeno de la extinción de la megafiuna y el posterior agravamiento de la desertificación como consecuencia def recalentamiento del clima, reducirán drísticamente esta posibilidad (Martin 1973, Irwin Williams y Haynes 1970). 


\section{LA INTERPRETACIÓN DEL PROCESO DE POBLAMIENTO}

Estos son, en función de la evidencia actualmeate disponible, los principales hechos relativos at primer poblamiento de América y, pese a su caráter fragmentario, es necesario intentar una interpretación antropológica de los mismos.

El proceso parece dividirse en dos grandes etapas y corresponder a dos modelos de interpretación. El primero referido a la ocupación de las estepes heladas de Siberia, Beringia y Alaska; el segundo, a partir de la llegada del hombre a las grandes praderas del centro-sur del Canadá y desde alli hacia el sur hasta llegar a Tierra del Fuego.

En la primer etapa las pautas de comportamiento cultural deberian ser la continuación de las del palcolítico, anteriormente descriptas, en las que el progreso tecnológico permite la ocupación de territorios cada vez más hostiles. La tasa de progresión debe haberse visto limitada por un difícil balance cutre avanzar en territorio hostil y desconocido, presionados por la búsqueda de un nuevo equilibrio demegráfico, o permanecer en el territorio conocido y desarrollar nuevas tecnologías que permitan afrontar las probables penurias de recursos.

La posibilidad de reconstruir estos modelos prehistóricas en base a los datos extraídos de las sociedades de cazadores-recolectores contemporáneos es bastante limitada pues, a los límites tradicionales para la extrapolación de datos a partir de estas sociedades (registros sincrónicos sin desarrollo temporal y carácter marginal de las mismas), se agrega ahora el problema de la existencia de una frontera abierta y, consecuentemente, de la faltir o debilidad de los limites impuestos al comportamiento de estas sociedades.

Deberemos pues intentar reconstruir los hechos a partir del testimonio arqueológico no obstante lo fragmentario de este,

Un aspecto notable es el diferente ritmo entre la ctapa nórdica del proceso y la colonización de las tierras templadas y cálidas que resulta de los datos disponibles.

La colonización desde Siberia a Alaska parece haber seguido un ritmo relativamente lento y constante en su camino hacia el este. La información de que disponemos sobre las estruturas identificadas en el sitio de Ushki (ver supra p. 24) reflejaria la existencia de agrupamentos 
con una gran cohesión social. Esta se infiere a partir de los restos de abrigos artificiales, construidos con pieles, piedras y huesos de mamut, que albergaban at un número indeterminado de individuos, subdivididos posiblemente en familias nucleares. Esto se desprende de la existencia de numerosos pequeños fogones al interior de estas grandes estructuras.

Lamentablemente estos testimonios son excepcionales, estando identificados, además del sitio de Ushai en Kamchatka, en el sitio de Molodova en Ucrania (Schovkoplyas 1971). Su existencia en ambos extremos del registro arqueológico de las estepas heladas, tanto del punto de vista cronológica como geográfico, podría estar indicando un rasgo característico y permanente de la colonización de las zonas frías.

Es posible que estas estructuras sean el reflejo de lazos sociales fuertes impuestos posiblemente por la hostilidad de un medio donde el número de individuos seria un factor clave, determinante, para asegurar la supervivencia. De ser así, las estructuras culturales que asegurarfan dichos lazos, habrán actuado, muy probablemente, de freno para la disgregación de estos grupos y por ende, para un desarrollo rípido del proceso de ocupación de los territorios vacios.

Pensemos también que estos pobladores peri-árticos contaban con posibilidades técnicas de acumulación de reservas conservadas por congelamiento, de las que no podrán disponer naturalmente sus descendientes de las zonas templadas. Atenuarian así la agudeza de los períodos de escasez de animales, consolidando esta estructura "conservadora" (Testart 1982). Tal aspecto babria variado sustancialmente con el avance en las zonas templadas, cada vez más benignas.

Hemos visto que en la mayoría de los casos, los asentamientos correspondientes a los primeros pobladores de América tienen un carácter esporádico, indicado por la escasa cantidad de vestigios alli encontrados. Por otra parte, la seleción de grandes animales como presas de caza, nos estaría indicando - por las dimensiones que dícba empresa requiere - que el nùmero mínimo de individuos de estos grupos no podía ser muy reducido. A su vez, el carácter vertiginoso del poblamiento del Canadá a Tierra del Fuego, de sólo 700 años, sólo puede explicarse por un importante crecimiento demogrăfico.

La economia cazadora, que conserva las pautas culturales de las regiones frias, puedes también haber generado mecanismos indirectos que ayuden a explicar el proceso de rápida ocupación del territorio. Es el 
caso del posible desbalance entre una dicta rica en grasas y un medio donde los requerimientos de calorías se han reducido, que pudo generar consecuencias a nivel de las tasas de fertilidad.

La base para la interpretación de esta segunda etapa del proceso radica en la dependencia entre los hombres y la fauna de grandes herbivaros (Kelly y Todd: 233-4).

Si el poblamiento se hubiera hecho en función del aprovechamiento de los diversos recursos que los nuevos medios geográficos iban poniendo a disposición de los hombres, este hubiera tenido un ritmo mucho más lento por el necesario aprendizaje.

A su vez, esta diversidad de recursos tendría necesariamente que reflejarse en una variación en el registro arqueológico a nivel de los instrumentos de piedra tallada. Esta diversidad no existe entonces y sí existe algunos siglos después, cuando la colonización ha sido completada y los distintos grupos tienen que conformarse con los recursos del lugar donde se encuentran.

La dependencia de los grandes herbivoros, sean estos Mamuts, Bisontes o Caballos, independizo a los grupos de un medio concreto y les permitió - amparados por la abundancia - multiplicarse y ocupar rápidamente nuevos territorios.

Hay dos interpretaciones principales sobre la forma en que se dío csta rápida expansión.

En primer lugar la de Paul Martin (1973), en la que se subraya el dificil equilibrio entre la megafauna pleistocena y el hombre.

Martín plantea que habría existido un frente de poblamiento, con alta densidad demográfica, en la zona de contacto entre los hombres y los nuevos territorios poblados de grandes animales. Esta diferencia de densidad entre las poblaciones humanas y las animales traeria como consecuencia la extinción de los grandes animales en esa zona - por la caza sistemática de que fueron objeto $-y$ por onde la necesidad de avanzar bacia nuevos territorios,

A su vez, la demografia explosiva se explica como consecuencia de la abundancia de recursos y de la potencialidad de la especie humana para multiplicarse cuando las condiciones le son favorables. El avanced del frente va dejando tras de si territorios donde la megafauna ya se ha extinguido y donde (faltos de recursos importantes inmediatos) permanecen los hombres en una muy baja densidad de población. 
Hay una scgunda interpretación - sostenida por Kelly y Todd (1988) que, partiendo siempre de la dependencia entre poblaciones humanas y megafauna, hace hincapié en los aspectos culturales de esta dependencia y no en el fenómeno de la extinción para explicar el carácter vertiginoso del poblamiento.

De acuerdo a esta hipótesis lo que explica el rảpido desplazamiento de los primeros pobladores es su capacidad (como to envidencia el registro arqueológico a través de instrumentos específicos y restos 6́seos) de cazar grandes mamíferos. Esta base de subsistencia no implica, en términos generales, la adaptación a ecosistemas especificos, sino que puede ser transladada a todos los medios donde existan grandes herbivoros en cantidad suficiente.

Los primeros pobladores se habrían desplazado constantemente tras los grandes animales. Dejarían asi pocos vestigios, impulsados por la retroalimentación de un sistema de subsistencia que los obligaba a buscar de forma permaneate zonas donde las densidades de caza fueren suficientes para mantener un modo de vida esencialmente cazador. No tienen el tiempo suficiente para realizar el aprendizaje de los otros recursos que los nuevos ecosistemas encontrados ponen a su disposición.

Esta teoria no es necesariamente contradictoria con la de Paul Martin aunque explica la baja densidad de restos arqueológicos y su gran dispersión geográfica, sin recurrir aecesariamente a un "frete" de alta densidad de pobladores. No rompe por lo tanto, abruptamente, con las pautas culturales-demográficas de la etapa anterior.

Para finalizar señalemos que tanto demográfica como socialmente (en el sentido de la necesidad de un número de grupos mínimo para construir un tejido social capaz de mantenerse y reproducirse) (Wobst 1976) estas perfomances de expansión en el tiempo y el espacio son perfectamente posibles $\sin$ forzar los datos sobre la capacidad demográfica de las poblaciones primitivas (Dumond 1975).

\section{LOS SITIOS SUPUESTAMENTE MAS ANTIGUOS Y SU DISCUSIÓN}

Es evidente que el esquema del poblamiento de América y los modelos de interpretación aquí presentados perderian automáticamento 
su valor si se demuestra que la llegada del hombre el Nuevo Mundo es muy anterior a 12 mil años, $y$ si las caracteristicas arqueológicas de esos sitios no permiten interpretarlos como de poblaciones esencialmente cazadoras. Debemos por lo tanto detenernos (brevemente por falta de espacio) en el problema de la existencia de una decena de sitios arqueológicos que pretendidamente reûnen ambas características (Guidon y Delibrias 1985, Ardila y Politis 1989).

Los dividircmos en dos categorías: 1) aquellos completamente atipicos en relación al modelo aquá planteado y 2) aquellos que pueden ser considerados sblo parcialmente contradictorios.

En el primer caso tenemos todos los sitios que reclaman una gran antigutedad y/o conjuntos ergologicos calificados de "toscos", "primitivos", "pobres", o "atípicos", con marcada preferencia por la utilización de materias primas locales (generalmente cuarzos y cuarcitas). Estas son de bajas performances en relación a la talla, y deben contraponerse con la búsqueda sistemática de finas rocas criptocristalinas por parte de los ocupantes de los sitios posteriores.

Estas industrias se pueden calificar tipológicamente como similares a las del paleolítico inferior y no pueden indentificarse como de gentes especializadas en la caza de la megafauna.

Estos sitios se encuentran todos en Sudamérica (a excepción del sítio de Calico, con 150 mil aĥos de antigutedad, en California, Leakey y otros 1972). Ordenados cronológicamente, partiendo de la mayor antigûedad, los principales - que han sido seleccionados por la calidad de las investigaciones realizadas, pero no los únicos - son los siguientes: Toca da Esperança, en el estado de Bahía, Brasil, con 300 mil años de antigủedad; Boqueirão da Pedra Furada, estado del Piaui, Brasil con por lo menos - 45 mil años; Monte Verde, en el centro-sur de Chile, con dos niveles de ocupación, uno de $33 \mathrm{mil}$ años y otro, de 13 mil ańos; Pikimachay en Ayacucho, Perú con fechados desde los 21 mil años de antigüedad (la información sobre todos estos sítios está sintetizada en Guidon y Delibrias 1985).

Los cuestionamientos que han impedido la validación de estos datos como hechos arqueologicos se centran en la determinación del carácter artificial o natural de los vestigios encontrados. Es decir, si estamos frente a artefactos, o si, por el contrario, se trata de objetos resultantes de la acción de la naturaleza. Estos últimos, en la literatura arqueológica, han 
sido descritos como "geofactos", "eolitos" 0 "naturifactos" (Haynes 1988, Oakley 1968).

Este tipo de pseudo-artefactos son relativamente frecuentes en aquellos contextos geologicos donde rocas duras, de fractura concoidal, han sido sometidas a golpes - torrentes, playas, etc. - o a fuertes presiones - desplazamiento de terrenos, glaciares, etc - Se prouce asi un lascado que da como resultado objetos de forma similar a algunos de los instrumentos de piedra tallada producidos por el hombre.

Los geofactos no presentan problemas cuando son encontrados en terrenos anteriores al pleistoceno, pero su interpretación se complica cuando el ballazgo se produce en contextos donde no puede excluirse la contemporaneidad de la presencia humana. De esta posibilidad resultan dos interpretaciones; una es la utilización directa de geofactos por el hombre en la medida que estos pueden cumprir funciones similares a tas de los verdadeiros artefactos y otra, la mís frecuente, su identificación errónea como artefatos (Bednarik 1989, Lynch 1990).

Los investigadores de los sitios arriba mencionados hacen hincapic en el carácter primitivo y atípico de las industrias por ellos encontradas, afirmando que quienes no reconocen los objetos propuestos como artefatos lo hacen en función de prejuicios derivados fundamentalmente de una (según ellos) trracional necesidad de vincular tipológicameate estos hallazgos con los de la posterior industria Clovis.

No podemos abundar aquí sobre los argumentos esgrimidos por una y otra parte; sé̉alemos que la polémica no se limita únicamente al origen antrópico de los objetos encontrados, sino que se refiere también a la correcta identificación de otras estructuras arqueológicas, como fogones y estructuras de madera, y a la interpretación estratigráfica de los sitios (Lynch 1990 y 1991, Guidon 1989, Dillehay y Collins 1991, Gruhn y Bryan 1991).

La segunda categoria corresponde a los sitios que estarian fechados en sólo algunos milenios más que los aquí aceptados. Tipológieamente pueden ser identificados como del paleolítico superior y/o de alguna facie de transición a este, pero siempre formando conjuntos que en contraposición a los anteriores pueden ser catalogados como "normales" o "típicos", y donde ha desaparecido esa preferencia por materias primas bastas. 
Los sitios de esta categoria, que a nuestro juicio son los más importantes en esta polémica, son: Meadowcroft, en el Estado de Pennsylvania en EEUU (Adovasio y otros 1978); los sitios de la región de El Jobo en el Estado de Falcón en Veneruela (Cruxent 1970) y el sitio de Los Toldos en la provincia de Santa Cruz en Argentina (Cardich y otros 1973).

En Meadowcroft se destacan, como elementos contrapuestos a la tesis aquí desarrollada, los fechados que podrian remontarse hasta hace 19 mil años y ta ausencia de puntas de proyectil acanaladas. En El Jobo hay fechados entre 13 y 14 mil ańos - cnSudamérica - con una tradición original de puntas de proyectil fusiformes. En Los Toldos, existe un fechado de 12 mil años en el extremo sur de América del Sur, y un conjunto ergológico definido por la ausencia de puntas de proyectil bifaciales.

En estos casos no hay lógicamente discusión sobre el carácter antrópico de los artefatos encontrados (por cjemplo puntas de proyectil talladas bifacialmeate) y lo que se pone en cuestión es el correcto fechado de los mismos.

Los cuestionamientos se refieren principalmente a la validez de las muestras tomadas para realizar los fechados de Carbono 14 , sea porque estas puedan estar contaminadas o porque su asociación con los vestigios fechados no pueda ser determinada fehacientemente.

Se critica tambien la asignación de determinadas fechas para niveles que no están estratigráficamente establecidos y cuyos fechados deberian ser evaluados en relación a los de los supuestos niveles anteriores del yacimiento (Lynch 1990: 22-3).

\section{COHERENCIA ARQUEOLOGICA Y COHERENCIA PREHISTORICA}

Es indudable que la revisión crítica de la problemática de estos sitios arqueológicos no puede ser hecha en el limitado espacio de este articulo, pero sí es importante subrayar el mareo conceptual dentro del cual debe ser encarada. Este puede ser dividido en dos planos sin perder de vista que ambos están permanentemente interrelacionados. 
El primer piano se refiere a la problemática estrictamente arqueológica, es decir, a los aspectos técnicos y metodológicos que permiten establecer hechos cientificos. En otras palabras, a la calidad y pertinencia de los datos arqueológicos que resultan de las investigaciones.

El segundo plano se refiere al marco general de interpretación prehistórica en el cual los datos alcanzados deben integrarse. Aunque es obvio, recordemos la preeminencia del hecho cientifico, del dato comprobado, sobre el marco interpretativo que deberá modificarse cada vez que entre en contradicción con estos.

En relación al primer plano ef tema central es la validación, la comprobación, la aceptación de un dato surgido en el muy particular contexto de la investigación arqueológica, La arqueologia no sólo no es una ciencia experimental, sino que en el curso de la investigación destruye, o modifica profundamente, al sujeito de estudio.

De este modo la aceptación de un dato resultante de una investigación arqueologica se basa en la calidad de la presentación: curriculum del investigador(es), descripciones, muestras, material gráfico y fotográfico, etc. y, fundamentalmente, de la repetición de hallazgos similares que actúa como sustituto de la duplicación experimental.

En lo que respecta al plano de la interpretacion este se construye a medida que se van confirmando hachos arqueológicos. Su pertinencia se va afirmando por la acumulación de datos, aunque siempre es posible que existan varias interpretaciones para un mismo conjunto de hechos en función de modelos teóricos distintes.

A medida que una abrumadora acumulación de datos va confirmando determinadas interpretaciones es lógico que, frente a la aparición de hechos arqueológicos que plantean un radical cuestionamiento a los modelos vigentes, estos seau minuciosamente analizados y evaluados en el plano que definimos como de coherencia arqueológica.

El "doble patrón de pruebas" (Bray 1988, Meltzer 1989) (uno riguroso para los sitios antigos y otro más benévolo para los recientes) contra el que claman los defensores de un poblamiento temprano, no es por lo tanto el resultado de algunat perversión del "establishment" de prebistoriadores con una supuesta actitud conservadora, sino que es el corolario normal de la dinámica del conocimiento cientifico en todas suas áreas (véase por ejemplo lo sucedido con la llamada "memoria del agua" que en su momento conmociono el mundo de la bioquimica). 
Paralelamente, esos nuevos hechos, aûn cuestionados, deben servir de base a un nuevo modelo de interpretación. Este nuevo modelo será tanto más aceptado en la medida que se integre a los modelos prehistóricos ya desarrollados. Pero sí este, yendo más allá del contexto de la Prehistória Americana, cuestiona los grandes marcos de referencia de la Prehistória Universal, su aceptación y consecuentemente, la de los datos que le sirven de base, sólo se barí frente a un importante conjunto de pruebas incuestionables.

Es ese marco interpretativo de la Prehistóna Universal el que abre profandas interrogantes sobre la coherencia de los hechos propuestos en los sitios de cronologia anterior a Clovis. Señalemos a titulo de ejemplo: ¿Cómo puede explicarse el desplazamiento de poblaciones por territorios helados provistas con un equipo técnico tan pobre? iPor qué dichas poblaciones no dejaron rastros en su pasaje por las zonas heladas del Viejo Mundo dónde el registro arqueológico de ocupación se remonta a lo sumo a 35 mil años? ¿Cuáles serian otras rutas alternativas? ¿Es acaso imaginable la navegación transoceánica por poblaciones pre-sapiens sapiens hace 300 mil o sólamente 60 mil años? ¿Por qué los pobladores de Quereo y Monte Verde se limitaron básicamente a utilizar guijarres en estado natural - o partidos por la naturalcza - cuando todo el comportamiento prehistórico de la bumanidad está caracterizado por la talla intencional de rocas duras? LPor qué los habitantes de Pedra Furada o de Pikimachay tallaron unicamente las toscas materias primas locales cuando disponian a corta distancia (como está demostrado por los niveles arqueológicos recientes de esos mismos sitios) de calcedonias con una mayor aptitud para la talla? ¿Qué sucede con los pobladores prehistóricos entre los 300 mil años de Toca da Esperanza y los quizás 60 mil del Piauí? ¿Porqué a lo largo de decenas de miles de años teremos una ocupación intensiva en Pedra Furada, o la extraordinaria reocupación de Monte Verde, y una ausencia total de yacimientos en el restos del continente? iPor qué a lo largo de decenas de miles de años no se encuentra ningún resto humano? y por ûltimo: ¿Por qué, pese a una búsqueda sistemática, no hay niveles pre-Clovis en los sitios de esta cultura

En resumen, son demasiadas preguntas que se plantean a datos seriamente cuestionados como para que podamos tomarlos en cuenta en un modelo de interpretación del poblamiento da América. 
Para finalizar con el capítulo de la coherencia recordemos que el problema del poblamiento tambićn ha sido abordado por otras disciplinas afines, como la antropologia biológica y la lingáística. De acuerdo a trabajos de sintesis recientes el modelo de interpretación que aquí propusimos no es contradictorio con los resultados obtenidos poe estas disciplinas (Meltzer 1989: 473-4). Digamos además, sin entrar en un campo que no nos compete, que las interpretaciones basadas en probables distancias genćticas entre las poblaciones ameriadias y las asiáticas que, de acuerdo a los "relojes biologicos" que se establezcan, suponen una mayor antiguedad que la aquí propuesta para la separación de estas poblaciones, sólo podrắn confirmarse en la medida que el hallargo arqueológico de restos humanos establezean la verdadera prehistoria de dicha separación. Al día de hoy, la antropología biológica no dispone de un sôlo diente anterior a la ocupación Clovis del continente.

\section{CONCLUSION}

Pese a que siguen planteadas un gran aúmero de incertidumbres los datos acumulados nos estarian permitiendo trazar un caadro coberente y quizás definitivo del primer poblamieato de América.

Este se habria producido como consecuencia de la evolución biologica y cultural del hombre quiea, ea la ulitima etapa del paleolitico, el llamado Paleolitico Superior que se iniciara unos 40 mil anios atrás, desarrolló la tecnología - y seguramente la organización social - que le permitio colonizar, a la ûltima frontera del Viejo Mundo, las estepas heladas del norte de Europa y de Asia.

La Prehistoria de dicha colonización se ha ido trazando poco a poco y de acuerdo a la misma, el hombre se encontraba en el extremo noreste de Asia, frente a América (entonces unida por tierra a Asia por la llamada Beringia), en el décimo quinto milenio antes del presente.

A partir de entonces comienza el primer problamiento de América, el que en su inf́cio contináa por regiones similares a las de Siberia en Alaska y Canada. Recién cobra un carácter original, americano, cuando estos pobladores descubren, trás obstáculos geográficos constituldos por glaciares, montañas y bosques, las tierras templadas, las grandes praderas 
pobladas por manadas de herbivoros de la llamada megafauna pleistocena.

Esta disposición, aparentemente ilimitada de recursos, permitirá a estos primeros habitantes modificar profundamente sus pautas culturales acelerando el proceso de colonización y generando en unos pocos siglos un "horizonte cultural" (tradición homogénea) que caracterizará a los primeros pobladores de la mayor parte de América,

Esta tradición se caracteriza por la adaptación a la cana de grandes herbivoros y el desarrollo consiguiente del equipo técnico necesario para ello, en el cual se destacan las puntas de proyectil talladas bifacialmente, notables por el "aflautado" o adelgazamiento de su base,

Estas caracteristicas culturales están documentadas en primer lugar en Norteamérica ea el décimo primer milenio antes del presente, donde reciben nombre de cultura Clovis.

Una vez ocupado todo el doble continente y extinguida la megafauna, la humanidad se irá asentando en los distintos ecosistemas comienza asi los distintos procesos de adaptación regional que caracterizado a la Prehistoria Americana.

\section{BIBLIOGRAFIA}

ADOVASIO, J. M.; GUNN, J.D.; DONAHUE, S. (1978). Progrem report on the Meadowerof rockshelter. A 16000 years chroeicie. Arinals New York Academy of Scieves: y, 288, p. 137-59,

AMEGHINO, F. (1906) Les formations sédimentaires du Crétact supéniuer et du Tesciaire de Patagonie, Anat Museo Nacion. Buesos Aires. v, XIV.

AMMERMAN, A. J. (1975) Late Pleiksoetne populetion dynamies: An altemative viow. Humen Ecology, v. 3, n, 4, p. 219.33.

ARDILA, G. y POL ITIS, G. (ISG) Nuevos datos para un nowvo problema. Miseo dd Oro, Bogota, n. 22, p. 346.

BEDNARIK, R, G. (1989) On the Pleistocese settlement of South Americs. Antipuity, v. 63, n. 238, p. 101-11.

BFITRAO, M. C y DANON, J. (1987) Evidence of lluman octupations during the Middle Pleitstocene at the Toca da Experança in Centril Arehueclogical Region, Siate of Bahis,

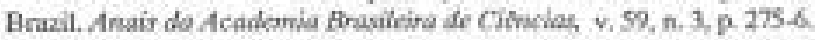

BNNORD, L. R. (1958) Et busta del Pasado, Critica. Befretona (1983).

BIRD, J, (1969) A comperison of South Chilean an Eruadorian "fishail" projectile points. Kracher Anzimpological Sociery Papers, n. 40, p. 52-71, 
BIRD, J. y COOKE, R. (1975) The ocurreace in Panuma of two types of Paloo-Indian projectile points En Earty Mar in Anericu from a Cincum-Pacific perpertive. A. Bryan (ed.). p. 263-72.

BORDES, F, \& THIBAULT; C (1977) Thoughes on the initial adaptation of Hominids to Europan Glacial Clinates. Qtearenay Reseonch, 3. 8. p. 1L5-27,

BRAY, W. (1988) The Palaeceindian debate, Natur, v, 312, p. 102.

BRYAN, A. (1928) An Overview of Paleo-American Prehiston from a Circum-pacific perspective. En Early Mar in America prow a Cincum-Pacific pospective. Edmoeton. Department of Anthropology, Usiversity of Alberta, A. Binan (ed.). p. 306-27.

BUDKO V. D. (1975) The Pelseolithic period of Byclorussia and adjoining aress. En 7he Origins of Houno sapien Unesco.

CANALS FRAU, S. (1973) Prehiworia de America, Buenos Aires, Sutamericana, (1950).

CARDICH, A. et aL (1973) Secuencia srqucológies de ia cueva 3 de Los Toldos (Santa Cruz,

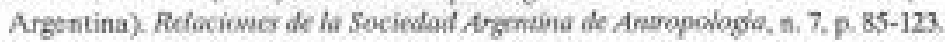

GARDICH, A. (1977) Las culturas Pleistocénicas y Postpleistocénicas de Los Tolbos (Santa Cruz, Argentina). En Tomo Cencriarie dé Musco de la Plowa, La Plata, 1 I Antrapoloria.

CHMIELEWSKJ, W., (1971) The costinuity and discontinwity of the evolution of a rehaeclogieal cultures in Cesinat and Eastem Europe between the S5ih and 25th millesaries B.C. Ea The Origis of Howio sopiess, UNESCO, p. 173.9.

COHEN, M. N. (1981) La crisis alimemaria ov la prefisionia. Madrid, Alianxs, (1977).

CRUXFNT, J. M. (1970) Projectile points with pleistocene mammals is Veneruela. Antipuigy, a. 44, p. 223.5.

DLAMOND, 3. M. (1957) Who were the first Americans? Nature, v. 329, p. 590-1.

DIKOV, N. N, y TTTOV, E. E. (1984) Prohlens of the stratification and periodization of the Ushki sitex. Anic Anihropowlowy, v. 22, n. 2, p. 69-80,

DILLEHAY, T. (1088) How sew is the New World? Andipaily, n. 62, p. 94.7.

DILLEHAY, T. D. y COLLING, M. B. (1991) Monte Verde, Chile: A comment on Lynch American Antriquin, y. 56, n. 2, p. 333-41.

DDXON, E. I. (1076) The pleistocene prehistory of Artic North Arserica, IX'e Coegr'es Union internationale des sciences préhistoriques et protohistoriques. Nice, Pp, 168-98.

DIXON, E. 3. (1985) Cultural chronology of Centeal interior Alaska. Arnc Arahopolog; v. 22. in. $1, \mathrm{p}, 47-66$.

DMITRIYEVA, I. N. (1980) Early Man in the Soviat Union, Cumen Anthopobog, v, 21. ก. 6. p. $828-30$.

DUMOND, D.E. (1975) The limitation of Humas Population: A Natura! History. Science, it. 18े7, p. $713-21$.

DUMOND, D. E. (1937) A reesamination of Eskimostleut Prehistdry. American Andso. pologsh, n. B9, p. $32-56$.

FLADMARK. K. R. (1979) Los pobladores de América. Murato Cienufico, v. 20 , n. 2. P. $1228-37$.

GERASAMOV, M. M. (1964) The Paloolithic site Malta: Excavations of 1956-57. Ent

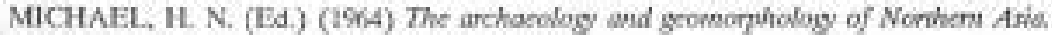
Torveto, Universiry of Toronto Press, o. 3-32. 
GRAHAM, J. A y HETZER, R. F. (196S) Man's antiquity in North Americx Views and facts. Quaremaria, n. 9, p. 225-35.

GRFENBERG. J; TURNER, C. G. y ZEOURA, L. (1966) The sestiement of the Americas;

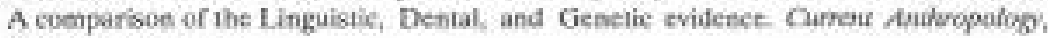
v. $27, \mathrm{n}, 5, \mathrm{p}, 477.97$.

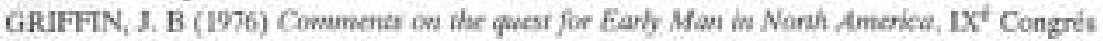
Laion Intermationale des Sciences Préhistariques et Protchistoriques. Nice, p. 8-17.

GRUIIN, R. y BRYAN, A. L. (1991) A revien of Lynch's descriptions of South American sites. American Antiquity, v. 56, n. 2, p. 341-7.

GUIDON, N, (1989) Da stratigraphly and chronology at Pedra Furada. Cumont Andhopology, v. 30, n. $5,9,647-2$.

GUIDON, N. y DEL IBRAS, G. (1955) Inventaire đes sites Sud-Antricains anterieurs a I2 000 anos. LiAnibingopologic, v. 89, n. 3, p. 385.408.

HAL.LAM, S. J. (1977) The relevance of Old Word Archaeology to the first eniry of man into New Warlds Colonizatioe seen fram the antipodes. Qmavemwy Rescranch, n, \&, p. $128,48$.

HAYNES, C. Y. (1966) Elephant huating in-North America. Eie Eurly man in Amorica:

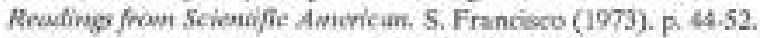

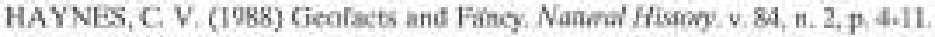

HAYDEN, A. (1981) Fesearch and developesent in the Stone Age: Technologieal Iransitions

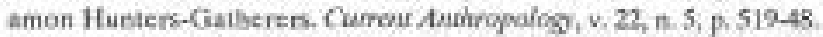

HOFFECKER, J. F, (1988) Applied geomorphology abd anchavological survey strategy for sites of Pleisacoene Age: An eximple from Central Alaska, Jowend of Archacologicol Stionct, 15: 683.713.

HOPFECKIR, J. F. (1989) Siberia. En Curreat Reserarch, T. Lynch. Anerican Antiquily, จ. $54, \mathrm{n}, 2,0.417-20$.

HOPKJNS et aL (Bus.) (1952) Paicoecaiby of Reringia New Youk, Academic Press.

HOWELL. F. C \& FREEMAN, L. G. (1\%2) Ambrona: An Earty Sione Age site on the Spanish Meseta. L. S. B. Leakcy Foudation News, n. 22, p. 11-13.

HRDLICKA, A, et al. (1012) Early man is .5outh America. Bureat of Amer. Ethnol. Sesithsonias Iastitutioa. Euell., it. 32, Washingos DC.

RWIN-WIL HAMS y HAYNES, C V. (1970) Cimatic thange and Early Population dynamics in the Soutbuestem United States, Qumoniary Reworch, v, 1, p. 59.71.

TMBEL.LONI, f. (1948) Tabala elasificatorie de los indios; ropiones biológieas y grupos raciales humanes de Aunética. Physis, p. $229-49,1 . X 11$.

IRWIN, H. T. y WORMtNGTON, H. M. (1970) Paleo-Indian sool byes in the Gre-at Plains.

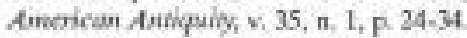

JAMES; S. R. (1959) Hominid use of fire in the Lower and Middle Pleistocene. Curnent Anthopolosy, \&., 30, n. 1, , . 1-26.

JEL.INEK, A. J. (1971) Early Man in tse New Worla: A techeological perspective. Artic Autherpolog, v, 8, i, 2, p. 15-2L.

KIE I.Y, R. L. Y TODD, 1. C (1968) Coming into the coutry Early Palcoindian hanting and mobility. Amoricent Arricesio, v. 53, n. 2, p. 231-44.

KI ELN, R, G. (t07]) The Fisistocene prehiblory of Siberia. Qwaveniary Research, n. 1, p 13361.

KLEIN, R. G. (1975) The reicuatce of Old Wors antheology to the first entry of Man into the New Woid. Quawemary Research, 4, 5, n, 3, p. 391-4 
K! FIN, R. 6, (1977) The ecolody of Earty Man is Soutern Africa. Science, x. 197, n. 4299, p. $115 \cdot 26$.

KRIBGER, A, (1964) Early man in the New World. En Prebivork men in the New World (de, J. Jennings y E Norbeck), Chicago, p. 23.81.

LAMING EMPERAIRE, A. (1990) le probidme des orisine amencaises. Théries, Hypotheses, documents, Lille.

LAUGHLIN, W:S. (1977) Origins and Aftinities of the First Americans Curen AnthopoLosy, v. 18, n. 3, p. 526-8.

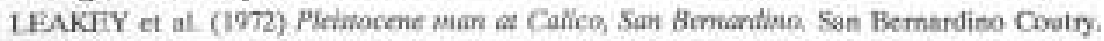
Muss. Ass.

LEE, R. B. (1981) is there a Poraging Mode of Production? Canadion fowmel of Anthupology, 4.2, n. 1, p. 13.9.

LYNCH, T. F. (1990) Glacial-Age Man in South Ameriea? A sritical revier. Americm Aritiquity, v. 55, a. 1, p. 12.36 .

LYNCH, T. F, (1991) Lack of evidence for Giacial-Age settlemeat of South Ameriea: Reply to Diliehry and Collins ant to Gruan and Hryan. American Amtignity, v. 56, y. 2, p. 349.55 .

MARTIN, P, S. (1973) The disconery of America, Science, v. 179, p, 969.74

MCBURNEY, C. B. M. (1971) Regional diflerenees in the dating of the earliest leptolithic Iraditicas. En The Origin of Home sapless. Unesoo, p. $237+\mathrm{n}$

MELTZIRR D. J. (1989) Why don't we know when the fiest peoplo came to North America? tonerican Astiquity, v, 54, a. 3, p, 47)-90.

MICHAPL, H. N. (Ed.) (19/4) The archaeology and gramarphoiogy of Nontem Asia. Toronso, University of Toronto Press.

MICHAEL H, N. (1984) Abouluie chrosologies of Late Pleisioecne and Early Haloecne cultures of Northeistern Asia Avic Anvimpology, $\vee, 21, n, 2, p .1-68$.

MOCHANOV, Y, (1978) Stratigraphy and atwolute chroeology of Northeast Asia, accoeding to the work of 196,-1933. En BRYAN, A. (Ed.) Early Man in Anerica from a Cincum Pacific perspective. Alberta. P. 54-63.

NAMI, H. G, (1987) Iaforme sobre la sezunda y tercera expedición a la Casva del Medio: Perspectivas aryqueofógicas para la Patagonia Austral. Anales del bnxituro de la Patagonia, Qile.

OAKI.EY, K. P. (1965) Man we noot-maker, Chicaga, The University of Chicago Press.

OKL ADNIKOV, A. P. (1961) The Paleolithic of Trans-Baikal.Amoricuen Antiquig, v. 26, n. 4. p. $486-97$.

OKI ADNIKOV, A. P, y POSPE.OOVA, G. A. (1982) Ulaliska, the oldest Palaedithic site in Siberia. Comour Anthopology, vi.23, n. 6, p. 710.2.

POWERS, W, y HOFTECKER, J. F. (1989) Late Pleistocene betthment in the Nenana vallg, Central Alaska. American Artiguiny, v. 54 n. 2, 5. 263-87.

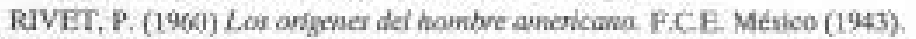

ROBERTS, P. H. H. (19\%) Developments in the problem of the North Ameriean

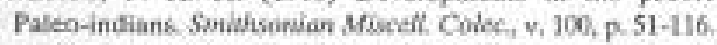

ROBFRTS, R. G. IONES, R. y SMTHH, M. A. (1900) Thermoluminescenet dating of a sopoo-year-0ld bumsen occupation site in northern Ausiralia. Nanine, n. 345, p. 153-6.

ROUSE 1. (1976) Peopling of the Americas. Qumomary Research, n. 6, p. 597-612. 
SCHOBtNGER, J. (1973) Nueves kallazges de puntas "Cola de Pescado" y consideracioses en torno al origem y dispersice de ls ewltara de Cazadores Superiores Toldense (Fell I) es Suramérica, XL. Congresso leternacionale degli Amecicanisth. Fuvens, v, L, p, 33-50,

SCHOBINCER, J. (1968) Prehistoria de Swounchicu 2. ed. Madrid, Alianza.

SCHOBINGER, 3, (1988b) Algunnas obsenveinots terminológicas nobre is Prehistoris

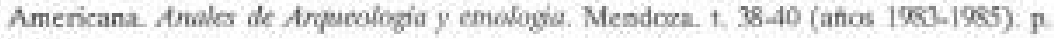
7.25 .

SCHOVKOPLYAS, L G. (1971) The Upper Paleolithic culture in the Utraine. En The Origin of Homo sopiens. Unesen. p. 181-6.

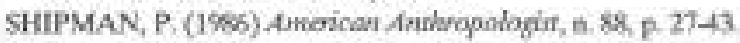

SPEIH, J. D. (1987) Les siratégies alimentaire des chasseur eveilleurs. La Recherche, v. 190, n. 18, p. 894.903.

TAIEB, M. \& POUPEAU, G. (1980) L'age du "prentier hiene". La Recherche, v. 1t, n. Ith p. 1314-5.

TEN BRINK, N. W. y WAYTHOMAS, C. F. (1995) Late Wisconsin Giacial chroeology of the North-Central Alaska range: A regional synthesis and its ittiplicatioes for Early Humat

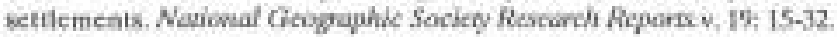

TESTART, A. (1982) The sigaificance of food storage among Hanter.Gatherems: Residence

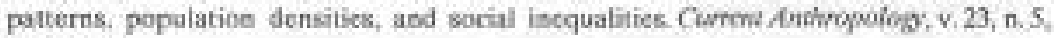
p. 523-37.

TOLSTOY, P, (1958) Te anchaeology of the Lena Basin an its New World relationahips, part Il. Amicrican Andipwity, v. 25, n. 1, p. 63-81.

VASKOVSKIY, A. P. (1964) A brief outline of the vegetation, elimate, and chinonolegy of the Quaternary period in the Upper Reaches of the Kolyma and Indigirka rivers and on the Northen ecast of the sea of Okhotsk. Fa MICHAFI, H. N. (Ed.) (1964) The ancheology and geomomphibiogy of Nondern Asia. Toroeto, Universily of Taronto Press, p. 464.505.

WATANABE, H. (1971) Periglacisl ecology and the emengence of Howo sapiens. En The Onigut of Hown sipiens. UNESCO, p. 271-85.

WHITE, T. D. Les Australopithèques. La Recherche, v, 13 n. 137, p. 1258.70 .

WoBsT, H. M. (1076) Locational relationnhips in Paboelithic Society. Jaumal of Hianon Evolucian, n. 5, p. 49.58. 